\title{
Dealing with the Blank in Books' Layout Design
}

\author{
Lu Liu \\ Department of Architecture and Art Design, Beijing Jiaotong University, Beijing 100044, China \\ E-mail: liujiazi21@sina.com
}

\begin{abstract}
In traditional Chinese painting, blank functions importantly. But it is different from that of a general sense. It is actually the organic component of the whole piece of art work. Blank has a rich expression, making pictures vivid and lively. In the traditional painting and modern layout design, the content is black, that is, the entity. However, it is the blank that is oversensitive. When every entity is put in the position, there must be some blank to adjust the visual intension so that the strain and dynamic can be released and extended. The virtual space is blank-leaving. The blank in the format is not only for the visual pleasure but for an aesthetic realm it creates for readers.
\end{abstract}

Keywords: Black, Blank, Visual spatial, Visual aesthetics

\section{The black and the blank create visual beauty}

In traditional Chinese painting, blank functions importantly. But it is different from that of a general sense. It is actually the organic component of the whole piece of art work. Blank has a rich performance, making pictures vivid and lively. Ancient Chinese once made summarization about the aesthetic forms, saying "model beauty depends on the modeling patterns outside of the images". In the traditional painting and modern layout design, the content is black, that is, the entity. However, it is the blank that is oversensitive. When every entity is put in the position, there must be some blank to adjust the visual intension so that the strain and dynamic can be released and extended. The virtual space is the blank-leaving. The blank in the format is not only for the visual pleasure but for an aesthetic realm it creates for readers. The artful blank in pursuit of blank beauty is helpful to foil the theme, create atmosphere, attract eyeballs, strengthen space levels and makes a clear and orderly layout with proper density. The blank in Chinese paintings becomes an integral element and helps create a rich imaginary world for the aesthetes. The blank in the format is actually a breathable visual space for readers to gain a happy visual experience in a relaxed state.

In general, the blank of book layout design is on the end paper between the cover and the main content. The large blank on the first page of a chapter gives a signal of a new content to the readers. There are page header and page footer on the text, left and right margins of the main content pages, and the blank in the last paper, etc. With the development and creation of the book's binding and layout design, these blanks left are getting more and more smart and fresh. Some design can give a large page header. Some can even get rid of the left margin while gives more space to the left margin. The flexible arrangement gives every book its own personality, even becomes a symbol of some book. The blank, which is also named the fourth element, is an important part in a book's layout. It helps create visual space for readers to feel the nimbus of a book in the traceless room. Many newspapers and magazines now have developed their own style of layout and techniques of blank creation. For example, VISION has large area in its content page. The big blank has lowered the rate of utilization and relieved the strong excitement of pictures and colors, showing unique cultural personality and taste. Many newspapers have layout design of blank around the header, in boot reading area and in the column spacing. The design of albums adopts blank as an important element. Proper blank-leaving can beautify the pattern making books look more natural and breathable. The more important is that blank-leaving has a unique guiding role. Although a page in a book or a text in a page can not possess the important position, the designer s can make use of blank-leaving to highlight that page or the content, thus producing the effect of attracting the readers' attention.

\section{The replacement of blackness and the blank}

The same as the painting principle emphasizes the configuration of virtual space, in which both the blank and the drawing are parts of the whole, the conversion of the blank and the black of the layout is actually a kind of expression. This blank-leaving can be space without any ink, and can also be an arbitrary color or decorative graphics. The use of soft grey is an approach to deal with the virtual space. The color of grey can soften the strong contrast of the white of blank and the black. Adopting the colors of black, white and grey harmoniously and the plane structure of point, line, and surface can make this text and that one, a text and its title, this illustration and that one become a unified organic whole. In a design, blank is used to be a modeling element because it is featured with images. Its form, size and proportion determine the quality and the capability of conveying the information of the layout. The blank and the entity 
depend on each other. The blank can make entity create the visual dynamic and gain the tension.

Excellent designers often gain their success by exploring and expressing the thoughts and feelings in what is designed. If a designer wants to successfully reproduce these thoughts and feelings, he should combine his emotion and design to create cleverly the blank and make full use of it to guide the readers' vision. Only by this way can he or she arrive at the creative thinking. In the process, the blank becomes the subject of the creation. It can be shielded and can be read by appreciation. The abstract of the blank has a virtual beauty, leaving space for further imagination of the scenery behind the veil. It is a form full of meanings and can deepen the information expressed in the design. It helps the readers to understand the work. Meanwhile it can create new visual expression and de-familiarize the work to attract the readers. In that case, the blank can be flexible and creative in the expressions. It can be the contrastive colors conflicting with the content of the layout, the pictures functioning as ingredients in editing, and the transitional part between entity and virtual scenes. The expression is often used in the fashion magazines. For example, a color mass integrated with the subject picture in the layout serves as the blank. But if it is taken out, it becomes independent with colors, form and expressive language. The expression of the form is different from that of Chinese paintings in which the most far-reaching pattern is empty (Lao Tze). The blank in layout, compared with that in Chinese paintings, has images and specific languages but what it has conveyed to the readers is the delight of exploring what is far to reach.

\section{Moderate blank-leaving}

The principal and the blank make an expression of rhythms and form aesthetic vision. However the blank can not be abused. In Chinese painting theory, it is said that dealing with the blank is much more difficult than drawing actual pictures. It needs careful consideration to deal with the blank in the layout design. Don't go to extremes by abusing the blank and making too much space. Too much blank or space may make the pattern look loose and make people feel empty and lifeless. The more important is that it is no good for reading. Some designers emphasize too much the artistry. They make the distance between the lines twice or three times than the spacing standard. And kerning is also too loose. Too big row spacing and kerning resulting in a big gap between lines and the unattached characters makes reading difficult. Others do not pay attention to the contrast relations expressed by the black and the white. They are extreme to use the contrast. Function should be the first to the designers in dealing with the relations between black and white. The practical function itself is one of the factors to produce beauty if it is reasonable. On the other side, too much blank in the layout will inevitably reduce the amount of texts and pictures, which is wasteful. This will undoubtedly increase the thickness of a book and make people feel that it can not provide readable information.

The moderation of blank-leaving must be considered carefully by the layout designers. Among different texts the blank should be left appropriately. It is necessary to separate articles to give readers a break, and keep the sense of the overall layout to form decent space. It is difficult to grasp the degree of blank-leaving. Do not fill where the blank should be left. Blank-leaving should make people feel neither empty nor boring.

Layout design is a process of artistic creation. A good layout can attract much attention from the readers, which is the prerequisite of effective communication. On the other hand, a good format can always participate in the performance of the book content. The layout design reflects the evaluation of designers to the content and its importance. In a layout, the designers should make use of the dynamic visual thinking to create the blank and skillfully adopting the blank-leaving and properly grasping the virtual space to produce a smooth and comfortable visual spatial effect with a coherent logic, the prioritized content, rich layers and the harmonious blank and black. So the readers can accept the information that the authors and editors want to convey while enjoying the beauty of the works, which is the ultimate goal of the layout.

\section{References}

Innovation in Book Design. (2007).Rotovision Book.

Brown, Miller. (2001). Design Feelings.

Davis, Graham. (2002). Shortcut to Layout. 09.

Fink,Gail-Deibler. (2002). White Design. 04. Shanghai People's Fine Arts Publishing House.

Harvey, Wilson. (2004). The Best of Brochure Design 07. Rockport Publishers.

Jiangcheng, Huang. and Yujun, Li. (2003). 08. Trip of Binding. Jiangxi Art Press.

Jingren, Lu. (2000). Jingren and His Books Design. Jilin Art Press.

Jingren, Lu. (2004). Open the Contemporary Chinese Books design. Tsinghua University Press.

Jingren, Lu. (2007). Book Games—40 designers in Contemporary China. Guangdong Nanfang Daily Press.

Supreme Form. Henan Literature Publishing House.Twemlow, Alice. (2007). Want is Graphic Design For? PRGEONE.

Visual performance. (2004). China Youth Publishing House. 01 